\title{
Vertical Strabismus and Positive Bielschowsky Head-Tilt Test: Atypical Presentation of a Maxillary Mucocele
}

\author{
Filipe André Correia ${ }^{1,2}$ Gustavo Filipe Antunes de Almeida ${ }^{1,2}$ Carolina Fernandes Pereira Bruxelas ${ }^{2,3}$ \\ Pedro Alberto Batista Brissos de Sousa Escada ${ }^{1,2}$
}

${ }^{1}$ Serviço de Otorrinolaringologia do Hospital de Egas Moniz, Centro Hospitalar de Lisboa Ocidental, Lisbon, Portugal

${ }^{2}$ Nova Medical School, Universidade Nova de Lisboa, Lisbon, Portugal

${ }^{3}$ Serviço de Oftalmologia do Hospital de Egas Moniz, Centro Hospitalar de Lisboa Ocidental, Lisbon, Portugal

Ann Otol Neurotol:2021;3:100-102

\author{
Address for correspondence Filipe André Correia, MD, \\ Otorrinolaringologia Centro Hospitalar de Lisboa Ocidental, \\ Rua da Junqueira 126, 1349-019 Lisboa, Portugal \\ (e-mail: filipe160490@gmail.com).
}

\begin{abstract}
Keywords

- vertical strabismus

- test of skew

- superior oblique muscle palsy

- skew deviation

- Parks-

Bielschowsky test

Test of skew has become a cornerstone in the approach of a patient with vestibular symptoms but a detected vertical misalignment may be caused by an oculomotor disturbance and not a skew deviation. We report the case of an elderly patient with a 1-month history of dizziness and visual disturbance that revealed on bedside examination a spontaneous left head-tilt and a pathologic alternate cover test, with right eye hypertropia and excyclotorsion, worse with right head-tilt. Dizziness was assumed to have a visual origin with unrecognized binocular diplopia, caused by an acquired right eye superior oblique muscle palsy. However, imaging revealed a right maxillary mucocele that eroded the orbit floor into the orbit. The change of the intraorbital component of the maxillary fluid-filled mass with head-tilt through a dehiscent orbital floor may explain the findings of vertical strabismus and positive Bielschowsky headtilt test in this case. Endoscopic treatment improved symptoms and findings.
\end{abstract}

\section{Introduction}

Test of skew has become a cornerstone in the approach of a patient with vestibular symptoms, since the advent of the three-step bedside oculomotor exam (H.I.N.T.S.) and due to its high sensitivity in detecting acute posterior fossae disorders causing dizziness. ${ }^{1}$ However, a vertical misalignment detected may be caused by an oculomotor disturbance and not a skew deviation. This clinical report reviews the diagnostic approach and explains the physiopathology of a positive Bielschowsky head-tilt test caused by a maxillary mucocele.

\section{Case Presentation}

Eighty-one-year-old patient presented to the emergency department, with a clinical picture of dizziness and unspecific visual disturbance that has started and remained stable over the past month. Past medical history included controlled arterial hypertension and sinus surgery years ago. Bedside examination revealed a pathologic alternate cover test, with right eye hypertropia, worse with right head-tilt. A spontaneous left head-tilt was noted. Right eye excyclotorsion was detected in the fundoscopic examination. Prism and alternate cover test established a vertical misalignment of 14, 26, 6 prism diopters
DOI https://doi.org/

$10.1055 / \mathrm{s}-0041-1724217$

ISSN 2581-9607.
(C)2021. Indian Society of Otology.

This is an open access article published by Thieme under the terms of the Creative Commons Attribution-NonDerivative-NonCommercial-License, permitting copying and reproduction so long as the original work is given appropriate credit. Contents may not be used for commercial purposes, or adapted, remixed, transformed or built upon. (https://creativecommons.org/licenses/by-nc-nd/4.0/).

Thieme Medical and Scientific Publishers Pvt. Ltd. A-12, 2nd Floor, Sector 2, Noida-201301 UP, India 

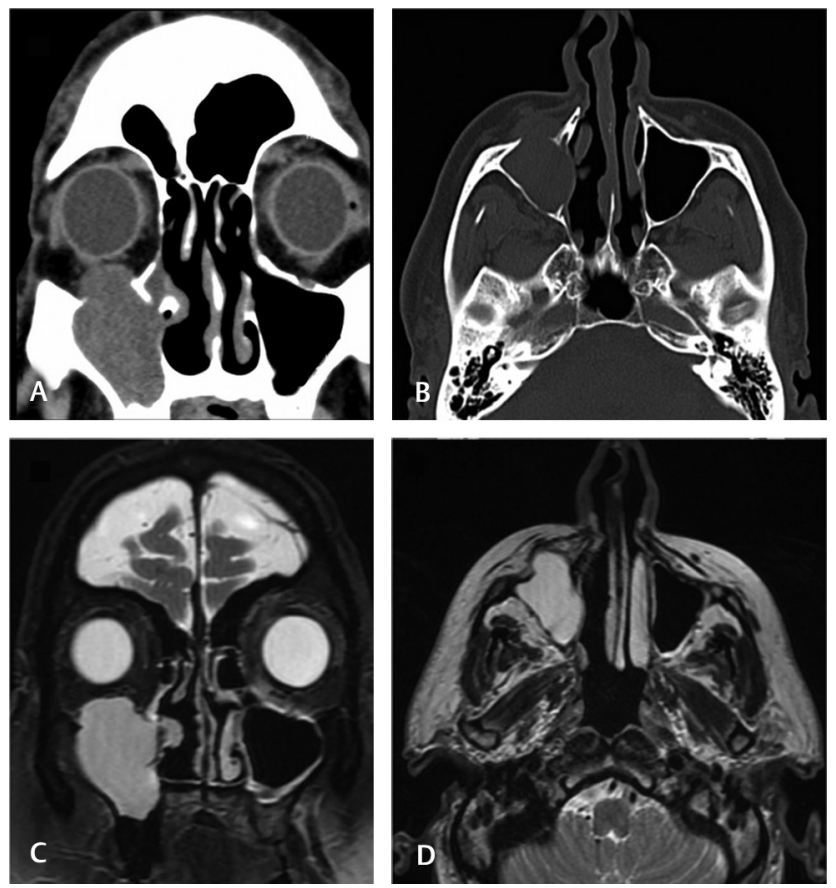

Fig. 1 (A) (coronal plane computed tomographic (CT) image; soft tissue window): expansive soft tissue density lesion originating in the right maxillary sinus and intraorbital component, causing right eye hypertropia. (B) (axial plane CT image; bone window): totally opacified right maxillary sinus; bulging of the lateral nasal wall; dehiscent anterior maxillary sinus wall; limited subcutaneous component of the homogeneous lesion; signs of inferior turbinectomy. (C) (coronal plane T2 magnetic resonance imaging (MRI) scan): hyperintense expansive maxillary lesion with intraorbital component that modulates inferior rectus and oblique muscles. (D) (axial T2 MRI scan): hyperintense lesion protruding through the dehiscent anterior sinus wall.

in centered, right, and left head-tilt positions, respectively. With no head-tilt, prism diopters were the same in right and left gaze. No other pathologic signs were detected.

Clinical history and physical examination, with right eye hypertropia and excyclotorsion and positive Bielschowsky head-tilt test, suggested an acquired fourth nerve palsy. Dizziness was assumed to have a visual origin with unrecognized binocular diplopia. The computed tomography (CT) scan showed a right eye subtle hypertropia, caused by a soft tissue density homogeneous mass with a maxillary sinus origin that eroded the orbit floor and had a relevant intraorbital component ( - Fig. $\mathbf{1 A}$ and $\mathbf{1 B}$ ). The magnetic resonance imaging (MRI) confirmed the mass as a maxillary mucocele ( - Fig. 1C and 1D). No posterior fossae disorders were detected on brain MRI. An endoscopic surgical approach to the mucocele was performed and dizziness and diplopia disappeared 1 week after treatment. -Fig. 2 displays three-step Parks-Bielschowsky test before and 1 week after surgery. After 1 year of follow-up, there was no recurrence of disease and the patient remained asymptomatic.

An informed consent has been signed by the patient, agreeing with the completion and dissemination of the findings and its results.

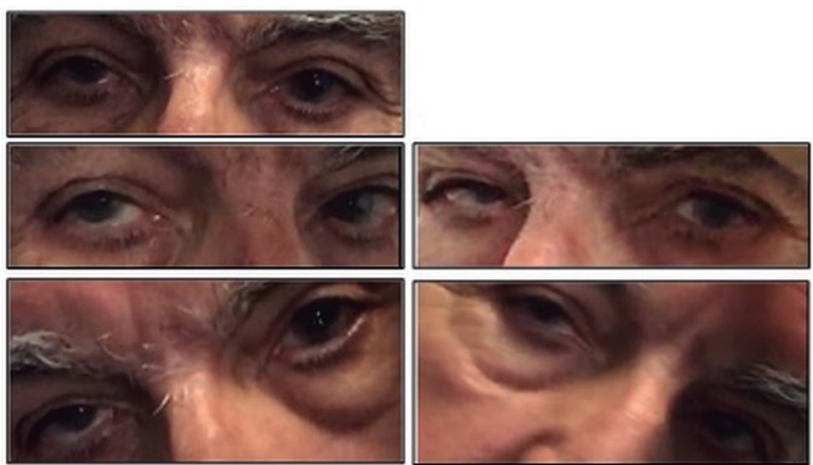

A
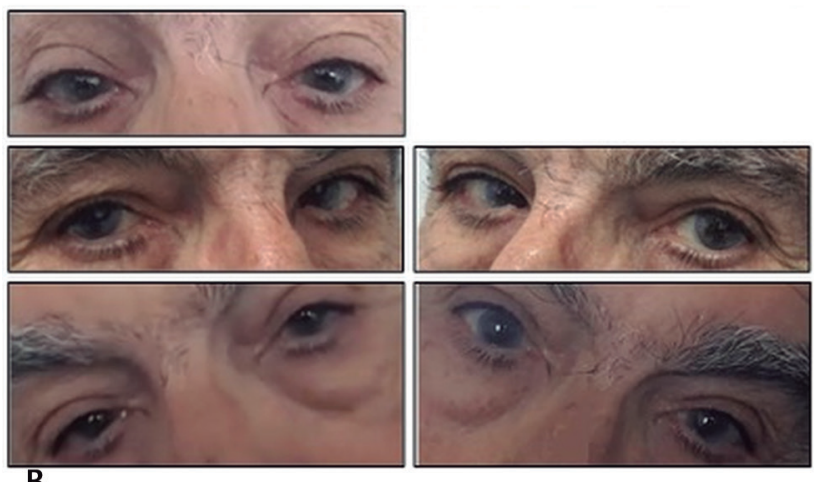

B

Fig. 2 Three-step Parks-Bielschowsky test. (A) Before surgery, right eye hypertropia worsened with right head tilt, but no change in right and left gaze was noticeable. Prism and alternate cover test (not shown) confirmed a vertical misalignment of 14, 26, 6 prism diopters in centered, right, and left head-tilt positions, respectively. (B) One week after surgery, no right eye hypertropia was detected.

\section{Discussion}

Vertical strabismus is an important sign to detect in the neurotology practice but differential diagnosis between fourth nerve palsy and skew deviation may be challenging. While skew deviation may represent an initial manifestation of a life-threatening brain stem stroke, an acquired isolated forth nerve palsy rarely represents an emergency. ParksBielschowsky three-step test ${ }^{2,3}$ has been described to differentiate them, but major drawbacks have been described. A positive Parks-Bielschowsky test, with increased hypertropia in contralateral gaze and ipsilateral head-tilt, while suggesting fourth nerve palsy, can also be present in patients with skew deviation. ${ }^{4,5}$ Besides, the complete three-step test fails to detect $30 \%$ of superior oblique muscle palsy cases, suggesting that a combination of a positive first and third steps may be more helpful. ${ }^{4}$ The upright-supine test, highly specific in chronic findings, ${ }^{6}$ has been proved unuseful in the acute and subacute context. ${ }^{7}$ In this setting, indirect ophthalmoscopy can help differentiating the two conditions, by measuring the cyclotorsion of the hypertropic eye: typically, the hypertropic eye is excyclotorted in fourth nerve paly and incyclotorted in skew deviation. ${ }^{8}$

In the reported case, the right eye excyclotorsion and hypertropia, evident on alternate cover test, increased with right head-tilt. The patient presented with a spontaneous left 
head-tilt and no other neurologic signs were detected. Thus, an acquired cyclovertical muscle palsy was suspected. In literature, there are some reports of extraocular muscle lesions after open and endoscopic sinus surgery, including cyclovertical muscle palsies. ${ }^{910}$ An acquired right superior oblique muscle palsy was considered and, as there was no history of recent trauma, ${ }^{11,12}$ the patient underwent brain and orbit CT and MRI scans, that showed a maxillary mucocele with an intraorbital component.

The distinguishing feature in this case was the positive Bielschowsky test. In a fourth nerve palsy, this sign is caused by a deficitary incyclotorsion of the superior oblique muscle during ocular counterrolling that is replaced by the action of the ipsilateral superior rectus, increasing hypertropia during ipsilateral head-tilt. ${ }^{2}$

The probable explanation of this case's positive Bielschowsky head-tilt test lies in the following facts: the intraorbital component caused a slight right eye hypertropia, mostly evident on alternate cover test; the inferior and medial pressure applied by the intraorbital mass to the right eye globe may explain excyclotorsion; with right head-tilt, the intraorbital component increased and the subsequent increase in pressure pushed the inferior surface of the right eye upward, increasing hypertropia; with left head-tilt, there was an increased pressure on lateral nasal wall, reducing hypertropia and eye torsion.

The patient referred no diplopia on left head-tilt, similar to symptoms relief with contralateral head-tilt in superior oblique muscle palsy; in both situations, spontaneous contralateral head-tilt is a compensatory mechanism to minimize the amount of their vertical strabismus and diplopia. ${ }^{3}$ Step 2 of Parks-Bielschowsky test did not reveal changes in right eye hypertropia because there was no change with gaze direction in the intraorbital volume of the mucocele and therefore in pressure. Normal superior oblique cross-sectional area on MRI and total resolution of symptoms and hypertropia after surgery confirmed the normal function of the extraocular muscles.

\section{Conclusion}

A maxillary fluid-filled mass may present with vertical strabismus and a positive Bielschowsky head-tilt test due to changes of the intraorbital component with head-tilt through a dehiscent orbital floor.

\section{Conflict of Interest}

None declared.

\section{References}

1 Newman-Toker DE, Kattah JC, Talkad AV., Wang DZ, Hsieh YH. H.I.N.T.S. to diagnose stroke in the acute vestibular syndromethree-step bedside oculomotor exam more sensitive than early MRI DWI. Stroke 2009;40(11):3504-3510

2 Parks MM. Isolated cyclovertical muscle palsy. AMA Arch Opthalmol 1958;60(6):1027-1035

3 Bielschowsky A. Lectures on motor anomalies. XI. Etiology, prognosis, and treatment of ocular paralyses. Am J Ophthalmol 1939;22(7):723-734

4 Manchandia AM, Demer JL. Sensitivity of the three-step test in diagnosis of superior oblique palsy. J AAPOS 2014;18(6): 567-571

5 Wong AMF. Understanding skew deviation and a new clinical test to differentiate it from trochlear nerve palsy. J AAPOS 2010;14(1):61-67

6 Wong AMF, Colpa L, Chandrakumar M. Ability of an upright-supine test to differentiate skew deviation from other vertical strabismus causes. Arch Ophthalmol 2011;129(12):1570-1575

7 Lemos J, Subei A, Sousa M, et al. Differentiating acute and subacute vertical strabismus using different head positions during the upright-supine test. JAMA Ophthalmol 2018; 136(4):322-328

8 Brandt T, Dieterich M. Skew deviation with ocular torsion: a vestibular brainstem sign of topographic diagnostic value. Ann Neurol 1993;33(5):528-534

9 Lin JS, Liu TT, Manes RP, Galvin JA. Superior oblique palsy: a complication of endoscopic sinus surgery. J AAPOS 2015;19(2): 180-181

10 Shapiro S, Schaefer JL, Gupta S, Nguyen J, Kellermeyer B. Isolated inferior rectus muscle entrapment following endoscopic sinus surgery. Case Rep Otolaryngol 2018;2018:4620510

11 Tamhankar MA, Kim JH, Ying GS, Volpe NJ. Adult hypertropia: a guide to diagnostic evaluation based on review of 300 patients. Eye (Lond) 2011;25(1):91-96

12 Diora JR, Plager DA. Sudden-onset trochlear nerve palsy: clinical characteristics and treatment implications. J AAPOS 2019;23(6):321.e1-321.e5 